NISSUNA UMANA INVESTIGAZIONE SI PUO DIMANDARE VERA SCIENZIA S'ESSA NON PASSA PER LE MATEMATICHE DIMOSTRAZIONI LEONARDO DA VINCI

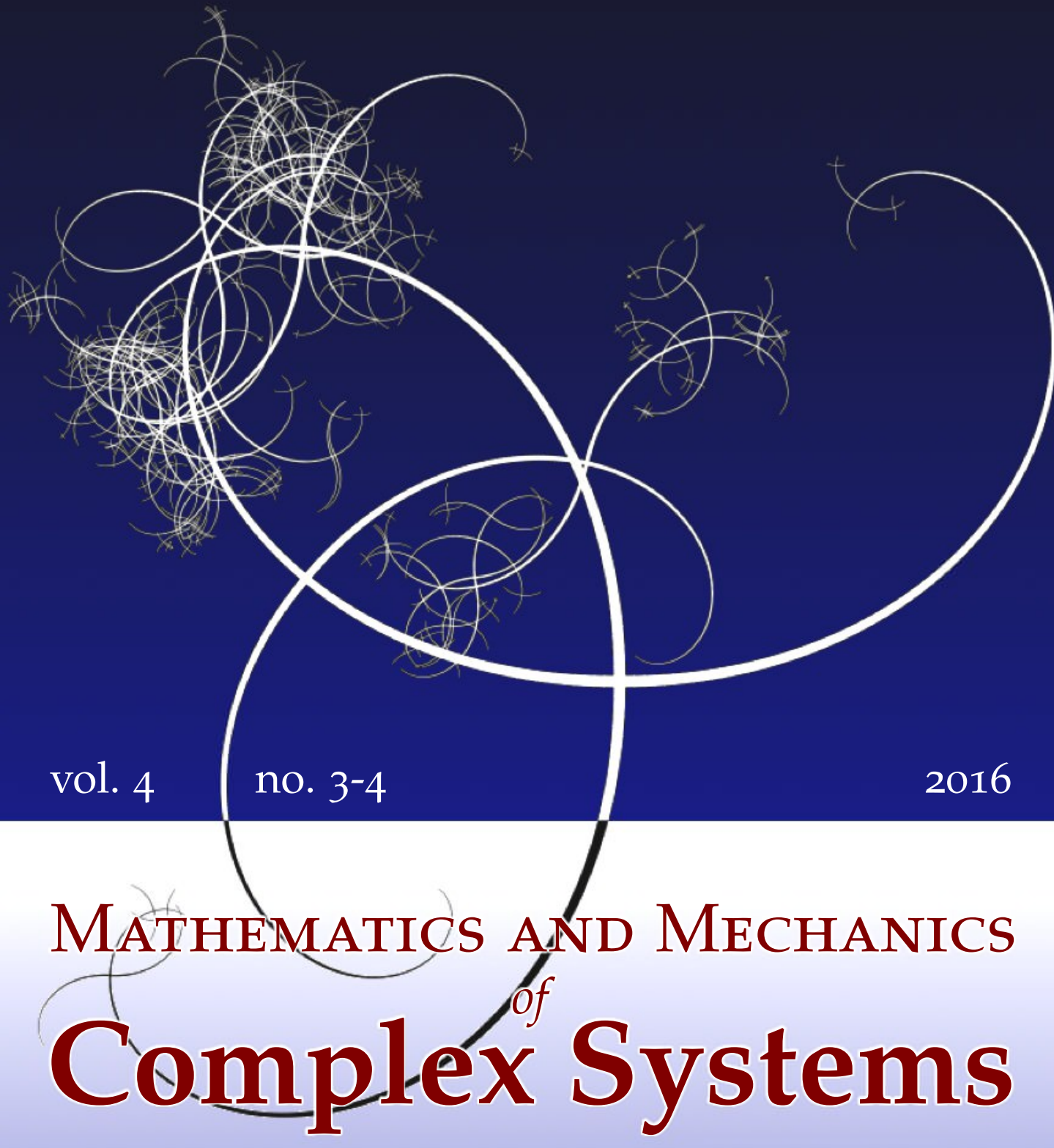

Rodolfo Figari AND Alessandro Teta

FROM QUANTUM TO CLASSICAL WORLD: EMERGENCE OF TRAJECTORIES IN A QUANTUM SYSTEM 


\title{
FROM QUANTUM TO CLASSICAL WORLD: EMERGENCE OF TRAJECTORIES IN A QUANTUM SYSTEM
}

\author{
Rodolfo Figari AND Alessandro TetA
}

\section{Dedicated to Lucio}

\begin{abstract}
This note deals with models of quantum systems where the emergence of a classical behavior can be concretely analyzed. We first briefly review some well known difficulties arising in the classical limit of quantum mechanics according to the Copenhagen interpretation. Then we discuss the seminal contribution by Mott (1929) on the tracks observed in a cloud chamber, where the problem can be approached in a particularly transparent way. Finally, we propose a model Hamiltonian, with interaction described by spin dependent point interactions, where Mott's analysis can be rephrased and the result can be rigorously formulated.
\end{abstract}

\section{Introduction}

Quantum mechanics is a theory of extraordinary success meant to describe the behavior of microscopic systems, i.e., systems with a typical action of the order of the Planck's constant $\hbar$. On the other hand, in the nonrelativistic regime, quantum mechanics is expected to be a universal theory and therefore should apply to the macroscopic world.

Its final formulation is due to Heisenberg, Born, Jordan [Born and Jordan 1925], Schrödinger [1982], Born [1926] and, for the mathematical aspects involved, to von Neumann [1932]. In fact, the quantum mechanical description of physical phenomena is rather abstract and counterintuitive, being based on the evolution of the wave function, i.e., a complex probability amplitude defined on the configuration space of the system with no direct physical meaning. This is in contrast with Newtonian mechanics, where physical objects are described through their positions and velocities, evolving in time in the phase space. The radically different approaches are the origin of some conceptual difficulties encountered when one tries to reconcile the two descriptions in some concrete physical situations. The aim of the present

\section{Communicated by Raffaele Esposito.}

PACS2010: 03.65.-w.

Keywords: environment induced decoherence, cloud chamber, transition quantum to classical. 
note is to discuss this delicate conceptual point through the analysis of concrete physical models. More precisely, the paper is organized as follows.

In this section we briefly review the basic rules of quantum mechanics in the realm of the so-called Copenhagen interpretation.

In Section 2 we analyze a specific case study where the problem of the emergence of a classical behavior in a quantum system appears in a clear way. In particular, we discuss the problem proposed by Mott [1929] concerning the emergence of a classical trajectory of a quantum particle in a cloud chamber.

In Section 3 we illustrate a model, based on a spin dependent point interaction Hamiltonian, where Mott's result can be rephrased in a more explicit and rigorous way.

Let us summarize the basic assumptions postulated for a system of $n$ quantum particles in $\mathbb{R}^{3}$. We avoid generality and technical difficulties and in particular we neglect the specific requests needed to describe systems of identical particles.

(1) State: The state of the system at time $t$ is described by the wave function $\psi_{t}\left(x_{1}, \ldots, x_{n}\right), x_{j} \in \mathbb{R}^{3}$, which is an element of the Hilbert space $L^{2}\left(\mathbb{R}^{3 n}\right)$ with $\left\|\psi_{t}\right\|=1$.

(2) Evolution of the state: Given the initial state $\psi_{0}$, the state at time $t$ is the solution of the Schrödinger equation

$$
i \hbar \frac{\partial \psi_{t}}{\partial t}=-\sum_{j=1}^{n} \frac{\hbar^{2}}{2 m_{j}} \Delta_{j} \psi_{t}+V\left(x_{1}, \ldots, x_{n}\right) \psi_{t},
$$

with initial datum $\psi_{0}$, where $m_{j}$ is the mass of the $j$-th particle, $\Delta_{j}$ denotes the Laplace operator relative to the coordinates of the $j$-th particle and $V$ is the interaction potential.

(3) Observables: An observable $\mathcal{A}$ relative to the system is represented by a selfadjoint operator $A$ in $L^{2}\left(\mathbb{R}^{3 n}\right)$. In a system made of a single quantum particle, simple examples of quantum observables are position and momentum. The position is represented by $\hat{x}_{k}$, defined as the multiplication operator by $x_{k}, k=1,2,3$, where $x_{k}$ denotes the $k$-th component of the position of the particle. Analogously, the momentum is represented by the differential operator

$$
\hat{p}_{k}=-i \hbar \frac{\partial}{\partial x_{k}}, \quad k=1,2,3 .
$$

One can easily check that the two observables do not commute and in fact, at least formally, they satisfy the Heisenberg commutation relations $\left[\hat{x}_{k}, \hat{p}_{l}\right]=i \hbar \delta_{k l} I$, where $I$ denotes the identity operator.

(4) Predictions: The predictions of the theory are given by Born's rule and, in general, are of probabilistic nature. In the special case of the position observable 
relative to a system made of one quantum particle, Born's rule reduces to

$$
\mathcal{P}(x \in \Omega ; \psi)=\int_{\Omega} \mathrm{d} x|\psi(x)|^{2},
$$

where the left hand side denotes the probability that the position of the particle described by the state $\psi$ is found in a set $\Omega \subset \mathbb{R}^{3}$. The prescription can be easily extended to the case of other observables making use of the spectral theorem for selfadjoint operators.

We list here few comments.

(i) As we already mentioned, the predictions of the theory are in excellent agreement with experiments. Furthermore, quantum mechanics is formulated as a universal theory (in the nonrelativistic regime) and therefore it can be used to describe both micro- and macrosystems.

(ii) Except in some special cases, Born's rule gives only probabilistic predictions. In particular, formula (1-2) means that the theory can only predict the statistical distribution of the detected positions in a large number of experiments made in identical conditions. We notice that a quantum particle, when a position measurement is performed in a single experiment, always appears localized in a well defined position which, in general, cannot be predicted by the theory. It should be emphasized that quantum mechanics is the first fundamental theory which explicitly renounces to a deterministic description of the physical phenomena.

(iii) For a single observable $\mathcal{A}$ represented by the operator $A$, let $\Delta_{\psi} A$ denote the mean square deviation of the statistical distribution of the possible values of $\mathcal{A}$ in the state $\psi$. It is always possible to find a state $\psi$ such that $\Delta_{\psi} A$ is arbitrarily small. This means that the values of the observable $\mathcal{A}$ in the state $\psi$ can be predicted with arbitrary accuracy. On the other hand, the noncommutative character of the algebra of observables implies that one cannot predict, with arbitrary accuracy, the value of all the observables relative to a given system. In particular, for a quantum particle in the state $\psi$ one can prove the Heisenberg uncertainty relations

$$
\Delta_{\psi} \hat{x}_{k} \Delta_{\psi} \hat{p}_{l} \geq \hbar / 2 \delta_{k l}
$$

It is evident that this inequality makes the notion of trajectory for a quantum particle problematic.

(iv) A crucial point of the theory is the linearity. This means that if $\psi_{1}(x)$ and $\psi_{2}(x)$ are two states then also the sum $\psi_{1}(x)+\psi_{2}(x)$, suitably normalized, is a possible state (superposition principle) and this property is preserved by the (linear) evolution. This apparently trivial fact has major physical consequences, because the predictions (see (1-2)) are given by a quadratic expression with respect to the 
state. In particular the probability density for the position is

$$
\left|\psi_{1}(x)+\psi_{2}(x)\right|^{2}=\left|\psi_{1}(x)\right|^{2}+\left|\psi_{2}(x)\right|^{2}+2 \operatorname{Re}\left(\psi_{1}(x) \overline{\psi_{2}(x)}\right) .
$$

From formula (1-4) it is clear that the situation described by $\psi_{1}(x)+\psi_{2}(x)$ cannot be considered in any sense as the "sum" of the situations described by $\psi_{1}(x)$ and $\psi_{2}(x)$ separately. In particular the last term in (1-4) is responsible for the appearance of interference effects, typical of waves, in the statistical distribution of the detected positions in a large number of identical experiments. Such effects can be directly observed in the so-called two-slit experiment.

We notice that a completely different situation occurs when we know that the system is in the state $\psi_{1}(x)$ with probability $p_{1}$ and in the state $\psi_{2}(x)$ with probability $p_{2}$, where $p_{1}+p_{2}=1$. In such a case the probability involved is due to our ignorance about the state and the system is described by a so-called classical statistical mixture of the two pure states $\psi_{1}(x)$ and $\psi_{2}(x)$.

(v) Another important aspect is the fact that the wave function is a "wave" in the configuration space of the system rather than in the three dimensional physical space. This implies the occurrence of "entanglement" for systems composed by more than one particle. An entangled state is a state that cannot be factorized in a product of one-particle states. At a kinematical level, this means that if a system is described by an entangled state $\psi\left(x_{1}, \ldots, x_{n}\right)$, it is not possible to associate a definite (pure) state to each subsystem. The situation is again radically different from the classical case and it is the origin of the "nonlocal effects" which can be produced on a subsystem $S_{1}$ acting on another spatially separated subsystem $S_{2}$.

We emphasize that there is a general and complete agreement in the physics community on the validity of the above rules, in the minimal formulation we have given. They are sufficient to give an accurate description of the physical phenomena and they can be considered the basis of the "pragmatic" view of the majority of the physicists working with quantum mechanics and without a specific interest in foundational problems.

On the other hand, one can strive for a better understanding of various ideas associated with the rules, such as the nature of the notion of probability arising in the theory, the meaning of an object's physical properties, the role of the measurement process and so on.

When one attempts to give an answer to such questions one enters the field of the so-called interpretational problem. Here, since the birth of quantum theory, many different views have been proposed that have stimulated a long and intense epistemological debate which is still active. We are not going to discuss here the different opinions and we limit ourselves to detail some aspect of the so-called standard or Copenhagen interpretation which, more or less consciously, is the point 
of view accepted by the majority of physicists. The aim is to highlight a conceptual difficulty arising in such an interpretation when one describes the measurement process or, more generally, the connection between the quantum and the classical description of the world.

The basic assumption in the Copenhagen interpretation is the completeness of the wave function. This means that the maximal information about a specific quantum system is encoded in its wave function. Therefore, the probabilistic predictions have an ontological character, i.e., they do not depend on our ignorance about some property of the system. If the system is in a state $\psi$ such that we cannot predict the value of an observable $\mathcal{A}$ with probability one, then the system does not have a definite, even if unknown, value of $\mathcal{A}$.

A consequence of this assumption makes the role of observation problematic. We recall that in classical physics a measurement of an observable is an innocuous process that reveals the value of the observable (which was already possessed by the system before the measurement). In the quantum case, and according to the Copenhagen interpretation, the situation is different. Let us assume that the system is in a state $\psi$ such that the observable $\mathcal{A}$ does not have a definite value. Assume that we perform a measurement of $\mathcal{A}$ and find a value $a_{0}$. If, after a very short time, we repeat the measurement of $\mathcal{A}$ we can reasonably expect to obtain the same value $a_{0}$. In other words, immediately after the first measurement we can predict with probability one the value $a_{0}$ for $\mathcal{A}$. This means that the measurement process is a strange mechanism that contributes to assign the value $a_{0}$ to the observable and it necessarily produces an instantaneous transition of the state $\psi \rightarrow \psi_{0}$, where $\psi_{0}$ is a state in which $\mathcal{A}$ has the definite value $a_{0}$ (typically an eigenvector of the self-adjoint operator $A$ representing $\mathcal{A}$ ).

The instantaneous (stochastic and nonlinear) transition $\psi \rightarrow \psi_{0}$ is called wave packet collapse.

Bohr [1928] explained the transition claiming that a measurement apparatus is a classical object and therefore it always possesses a definite value of its observables; he assumed that the interaction between the classical apparatus and the quantum system determines the collapse, inducing the transition of the quantum system state.

Bohr's explanation can be criticized in many respects. We simply mention the following observations.

It is not clear where the border line between the measurement apparatus (showing a classical behavior) and the system (showing a quantum behavior) should be fixed. The problem is usually solved pragmatically for each specific situation, but, at the conceptual level, there is ambiguity.

An even more relevant point is the fact that it is not explained why a measurement apparatus, despite being made of atoms, cannot be described by quantum 
mechanics and it is a priori considered as a classical object with well-defined classical properties.

On the other hand, taking a different point of view, one can insist to describe also the measurement apparatus as a quantum system using the Schrödinger equation. As a matter of fact, in this case one can arrive at the unpleasant situation in which the apparatus' pointer has no definite position. This fact, as clearly explained by von Neumann [1932], happens when the system is in a superposition state of two eigenstates of the observable to be measured. Due to the linearity of the evolution and to the interaction system-apparatus, such a superposition state is transferred to the pointer which, as a consequence, has no definite position after the measurement.

It should be underlined once again that the above difficulty arises only at a conceptual level. Roughly speaking, in concrete physical models describing the system-apparatus interaction the "difference" between the situation "the pointer has no definite position" and the situation "the pointer has a definite, even if possibly unknown, position" is so small that it is practically undetectable.

To summarize the situation, J. Bell [1987] wrote that the quantum description entirely based on the Schrödinger equation works perfectly well for all practical purposes (a phrase used often enough that he abbreviated it as FAPP), but he also insisted that a conceptual inconsistency in the Copenhagen interpretation of the formalism related to the measurement problem undoubtedly exists.

\section{Mott's analysis of the cloud chamber problem}

Here we briefly recall the basic principles of a cloud chamber [Leone and Robotti 2004; Gupta and Ghosh 1946] and the first theoretical explanation of the observed tracks proposed by Mott [1929]. For a more detailed historical analysis we refer to [Figari and Teta 2013; 2014].

The air contained in the cloud chamber, saturated with water vapor, is brought into a supersaturated state by means of a fast expansion that suddenly lowers its temperature. An $\alpha$-particle, released by a radioactive source in the center of the chamber, interacts with the atoms of the gas, inducing ionization. The ionized atoms then act as condensation nuclei, giving rise to the formation of small drops of water. The sequences of these drops form visible tracks which are naturally interpreted as magnifications of the $\alpha$-particle "trajectories". In fact, they are accurately characterized as trajectories of a charged classical particle (relativistic or nonrelativistic according to the initial particle velocity) in a classical electromagnetic field. And, in particular, they are straight lines whenever no electromagnetic field is present.

The first quantum theoretical analysis of the radioactive decay of a nucleus with the emission of an $\alpha$-particle was given by Gamow [1928] and by Condon and 
Gurney [1928]. The authors concluded that the initial state of the emitted $\alpha$-particle has to be described by a spherical wave, with center in the nucleus and isotropically propagating in space.

It was immediately noticed that the spherical shape of the initial state was apparently in contrast with the observed tracks in the cloud chamber. In particular, in the words of Mott [1929]: "it is a little difficult to picture how it is that an outgoing spherical wave can produce a straight track; we think intuitively that it should ionise atoms at random throughout space." In an attempt to obtain a satisfactory theoretical explanation of the tracks, it was realized that a crucial role must be played by an act of measurement responsible for the collapse of the spherical wave. This requires an establishment as to where a line of separation between the quantum system and the measuring device must be fixed. In this sense, the debate on the cloud chamber can be considered as the prototype of any further discussion about the measurement problem and the appearance of a classical behavior in a quantum system in the context of the Copenhagen interpretation of quantum mechanics.

In a first possible approach, the $\alpha$-particle is the quantum system under consideration and the gas of the chamber acts as the measurement device by which we observe the particle. Therefore, an atom of the gas "measures" the position of the $\alpha$-particle which must be considered a particle immediately after the disintegration process, since at that moment the gas (i.e., the device) reduces the initial spherical wave to a narrow wave packet with a definite momentum.

Another approach consists of considering the $\alpha$-particle and the gas as the whole quantum system under consideration. In this case, one observes the ionized atoms and the wave function $\psi$ of the system should provide the ionization probability.

According to this second point of view, the above mentioned intuitive difficulty can be overcome, since it arises from our erroneous "tendency to picture the wave as existing in ordinary three dimensional space, whereas we are really dealing with wave functions in multispace formed by the coordinates both of the $\alpha$-particle and of every atom in the Wilson chamber" [Mott 1929].

In his paper Mott proposes a simplified model to obtain a theoretical explanation following the second point of view. He considers a three particle system consisting of the $\alpha$-particle, initially described by a spherical wave centered at the origin, and two hydrogen atoms, initially in their ground state, whose nuclei are fixed at the positions $\boldsymbol{a}_{1}, \boldsymbol{a}_{2}$, with $\left|\boldsymbol{a}_{1}\right|<\left|\boldsymbol{a}_{2}\right|$. The $\alpha$-particle interacts with the electrons of the atoms while the repulsion between the two electrons is neglected. Moreover, he introduces some assumptions on the physical parameters characterizing the model: first, the observation point is far away from the origin and the atoms; secondly, the $\alpha$-particle has a high momentum and the collision with the atoms is almost elastic. Then he looks for a solution to the corresponding time-independent Schrödinger equation. Using perturbation theory up to second order and standard stationary 
phase arguments, he computes the probability that both atoms are excited. His important result can be formulated as follows: the probability that both atoms are excited is (nearly) zero unless $\boldsymbol{a}_{1}, \boldsymbol{a}_{2}$ and the origin lie on the same straight line.

Let us comment on the meaning of this statement.

Since the observed phenomenon in the experiment is the excitation of the atoms, Mott's result says that one can only observe straight tracks. Therefore, it provides a clear physical explanation of the straight tracks observed in the chamber entirely based on the Schrödinger equation, without any use of the collapse postulate. On the contrary, the explanation given following the first approach appears physically obscure, since it is not a priori clear how an atom (i.e., a microscopic system) can act as measurement device.

It should be stressed that the above result holds under specific physical assumptions on the parameters of the model. This means that the observed behavior of the $\alpha$-particle in a cloud chamber is far from being universal.

It is worth mentioning that Mott's analysis is the first example of an approach typical of the modern theory of environment-induced decoherence [Blanchard et al. 2000; Joos et al. 2003; Hornberger 2009; Adami et al. 2004; 2006]. In fact, the classical behavior (the trajectory) of the system ( $\alpha$-particle) emerges as an effect of the interaction with the environment (vapor atoms in the chamber) under suitable assumptions on the physical parameters of the model.

This approach is particularly interesting since it is based on a quantitative description of the phenomenon, with a possible explicit control of the approximations. This is a crucial aspect in a detailed comparison between theory and experiment, which certainly would not be possible following an approach based on the idea of wave packet reduction.

It should also be noted that from the mathematical point of view some aspects of Mott's analysis can be improved. For instance, the stationary phase theorem is used without an accurate control of the conditions of applicability. Another unsatisfactory aspect is the use of the stationary Schrödinger equation, which prevents a time-dependent description of the evolution of the whole system. The use of the time-dependent Schrödinger equation, with a clear definition of the initial state and an explicit description of the successive interactions of the $\alpha$-particle with the first and the second atom, is required to make both the analysis and the result more transparent. We refer to [Dell'Antonio et al. 2008; 2010; Finco and Teta 2011; Recchia and Teta 2014; Teta 2010] for results in this direction.

\section{A model of cloud chamber}

In this section we present a model of a quantum environment inside which a quantum particle evolves. The environment should mimic the supersaturated vapor of 
a cloud chamber where ionization takes place when a fast and massive particle is passing by. The model we propose consists of a quantum particle interacting via point interactions with localized finite-dimensional quantum subsystems (twolevel atoms or spins). Details of the construction of the Hamiltonian of the whole system can be found in [Cacciapuoti et al. 2007] (see also [Albeverio et al. 2005] for a comprehensive analysis of point interactions Hamiltonians).

We consider $N$ spins placed in fixed positions $\boldsymbol{y}_{1}, \boldsymbol{y}_{2}, \ldots, \boldsymbol{y}_{N}$. The state space of the system is the Hilbert space

$$
\mathcal{H}=L^{2}\left(\mathbb{R}^{3}\right) \otimes \mathbb{S}_{N} \equiv L^{2}\left(\mathbb{R}^{3}\right) \otimes\left(\mathbb{C}^{2}\right)_{1} \otimes \cdots \otimes\left(\mathbb{C}^{2}\right)_{N},
$$

where $\left(\mathbb{C}^{2}\right)_{j}$ denotes the $j$-th copy of $\mathbb{C}^{2}$ in which the $j$-th spin state is represented (note that the particle itself is assumed to have no spin). To describe the $j$-th spin state in $\left(\mathbb{C}^{2}\right)_{j}$ we take the basis made up of the first Pauli matrix eigenvectors

$$
\hat{\sigma}_{j}^{(1)} \chi_{\sigma_{j}}=\sigma_{j} \chi_{\sigma_{j}}, \quad \hat{\sigma}_{j}^{(1)}=\left(\begin{array}{rr}
1 & 0 \\
0 & -1
\end{array}\right), \quad \sigma_{j}= \pm 1 .
$$

Any state in $\mathcal{H}$ can be written according to the following decomposition

$$
\begin{gathered}
\Psi \in \mathcal{H}, \quad \Psi=\sum_{\sigma} \psi_{\sigma} \otimes \mathcal{X}_{\sigma}, \\
\sigma=\left\{\sigma_{1}, \sigma_{2}, \ldots, \sigma_{N}\right\}, \quad \mathcal{X}_{\sigma}=\chi_{\sigma_{1}} \otimes \chi_{\sigma_{2}} \otimes \cdots \otimes \chi_{\sigma_{N}},
\end{gathered}
$$

where $\psi_{\sigma} \in L^{2}\left(\mathbb{R}^{3}\right)$. Using such a decomposition, the scalar product in $\mathcal{H}$ reads

$$
\langle\Psi, \Phi\rangle=\sum_{\sigma}\left(\psi_{\sigma}, \phi_{\sigma}\right),
$$

where $(\cdot, \cdot)$ is the scalar product in $L^{2}\left(\mathbb{R}^{3}\right)$ and the induced norm will be denoted by $\|\cdot\|$. With this notation, the normalization condition in $\mathcal{H}$ reads

$$
\|\Psi\|_{\mathcal{H}}^{2}=\langle\Psi, \Psi\rangle=\sum_{\sigma}\left\|\psi_{\sigma}\right\|^{2}=1
$$

The Hamiltonian $H_{0}$ acting in $\mathcal{H}$ generating the free dynamics of the system reads

$$
\begin{aligned}
& D\left(H_{0}\right)=H^{2}\left(\mathbb{R}^{3}\right) \otimes \mathbb{S}_{N} \equiv H^{2}\left(\mathbb{R}^{3}\right) \otimes\left(\mathbb{C}^{2}\right)_{1} \otimes \cdots \otimes\left(\mathbb{C}^{2}\right)_{N}, \\
& H_{0}=-\frac{\hbar^{2}}{2 m} \Delta \otimes \mathbb{\rrbracket}_{\mathbb{S}_{N}}+\sum_{j=1}^{N} \rrbracket_{L^{2}} \otimes \alpha_{j} \mathbf{S}_{j}, \quad \alpha_{j} \in \mathbb{R}^{+}, \\
& \rrbracket_{\mathbb{S}_{N}}=\rrbracket_{\left(\mathbb{C}^{2}\right)_{1}} \otimes \cdots \otimes \rrbracket_{\left(\mathbb{C}^{2}\right)_{N}},
\end{aligned}
$$

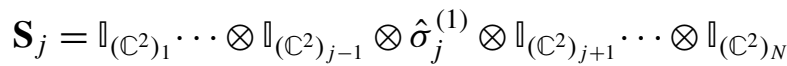

In (3-7), $\square_{\left(\mathbb{C}^{2}\right)_{j}}$ and $\square_{L^{2}}$ denote the identity operators in $\mathbb{C}^{2}$ and in $L^{2}\left(\mathbb{R}^{3}\right)$ respectively. The system energy is the sum of the kinetic energy of the particle and the energy of the spins. The $j$-th spin has an energy $\pm \alpha_{j}$ respectively in the states $\chi_{ \pm 1}$. In 
order to simplify notation, we will take in the following $\hbar=1,2 m=1$. The action of $H_{0}$ on functions of its domain is

$$
H_{0} \Psi=\sum_{\sigma}(-\Delta+\boldsymbol{\alpha} \cdot \boldsymbol{\sigma}) \psi_{\sigma} \otimes \mathcal{X}_{\sigma}
$$

where $\boldsymbol{\alpha}=\left(\alpha_{1}, \ldots, \alpha_{N}\right), \boldsymbol{\alpha} \cdot \boldsymbol{\sigma}=\alpha_{1} \sigma_{1}+\cdots+\alpha_{N} \sigma_{N}$.

The spectrum of $H_{0}$ is easily derived from the spectrum of the free Laplacian

$$
\sigma_{\mathrm{p}}\left(H_{0}\right)=\varnothing, \quad \sigma_{\mathrm{ess}}\left(H_{0}\right)=\sigma_{\mathrm{ac}}\left(H_{0}\right)=[\mu, \infty), \quad \mu=\min _{\boldsymbol{\sigma}}(\boldsymbol{\alpha} \cdot \boldsymbol{\sigma}) .
$$

Under the unitary group $e^{-i t H_{0}}$, the particle evolves freely with no interaction with the spins. The evolution of the $j$-th spin first component eigenstate corresponding to the eigenvalue \pm 1 is given by $e^{\mp i \alpha_{j} t} \chi_{ \pm 1}$. In fact, $e^{-i t H_{0}}$ is explicitly known and the solution of the Cauchy problem for the Schrödinger equation with initial datum $\Psi^{0}=\sum_{\sigma} \psi_{\sigma}^{0} \otimes \mathcal{X}_{\sigma} \in \mathcal{H}$ is

$$
\Psi^{t}=e^{-i t H_{0}} \Psi^{0}=\sum_{\sigma}\left(U^{t} \psi_{\sigma}^{0}\right) \otimes e^{-i \sigma \cdot \alpha t} \mathcal{X}_{\sigma},
$$

where

$$
\left(U^{t} f\right)(\boldsymbol{x})=\int_{\mathbb{R}^{3}} \mathrm{~d} \boldsymbol{x}^{\prime} \frac{e^{i\left|\boldsymbol{x}-\boldsymbol{x}^{\prime}\right|^{2} /(2 t)}}{(4 \pi i t)^{3 / 2}} f\left(\boldsymbol{x}^{\prime}\right)
$$

In order to define a Hamiltonian with spin-dependent point interactions placed at $\boldsymbol{y}_{1}, \ldots, \boldsymbol{y}_{N}$, one can start from the following consideration. Since the interaction must be nontrivial only at $\boldsymbol{y}_{1}, \ldots, \boldsymbol{y}_{N}$, such a Hamiltonian should act as the free Hamiltonian on wave functions vanishing at the points $\boldsymbol{y}_{1}, \ldots, \boldsymbol{y}_{N}$. This suggests considering the operator $\tilde{H}_{0}$ defined as the restriction of $H_{0}$ to the set of states whose spatial support does not contain $\boldsymbol{y}_{1}, \ldots, \boldsymbol{y}_{N}$ and to define the spin-dependent point interaction Hamiltonian any (nontrivial) self-adjoint extension of $\tilde{H}_{0}$.

In the following theorem we summarize the definition and properties of the Hamiltonians in the subfamily of the "local" self-adjoint extensions, characterized by local singular boundary conditions on each point $\boldsymbol{y}_{j}$. In order to state the theorem we introduce the notation

$$
\Phi_{j \sigma}^{z}=G^{z-\boldsymbol{\alpha} \cdot \boldsymbol{\sigma}}\left(\cdot-\boldsymbol{y}_{j}\right) \otimes \mathcal{X}_{\sigma}, \quad z \in \mathbb{C} \backslash \mathbb{R}
$$

where

$$
G^{w}(\boldsymbol{x})=\frac{e^{i \sqrt{w}|\boldsymbol{x}|}}{4 \pi|\boldsymbol{x}|}, \quad w \in \mathbb{C} \backslash \mathbb{R}^{+}, \quad \operatorname{Im} \sqrt{w}>0
$$

Then we have 
Theorem 3.1 [Cacciapuoti et al. 2007]. Define the operator $H^{A}$ with domain

$$
\begin{aligned}
D\left(H^{A}\right)=\left\{\Psi=\sum_{\sigma} \psi_{\sigma} \otimes \mathcal{X}_{\sigma} \in \mathcal{H} \mid \Psi=\Psi^{z}+\sum_{j \sigma} q_{j \sigma} \Phi_{j \sigma}^{z} ;\right. \\
\left.\Psi^{z} \in D\left(H_{0}\right), \operatorname{Im} \sqrt{z}>0, \sum_{j^{\prime} \boldsymbol{\sigma}^{\prime}} A_{j \sigma, j^{\prime} \boldsymbol{\sigma}^{\prime}} q_{j^{\prime} \boldsymbol{\sigma}^{\prime}}=f_{j \sigma}\right\},
\end{aligned}
$$

and action

$$
H^{A} \Psi=H_{0} \Psi^{z}+z \sum_{j, \sigma} q_{j \sigma} \Phi_{j \sigma}^{z}, \quad \Psi \in D\left(H^{A}\right),
$$

where

$$
\begin{aligned}
q_{j \sigma} & =\lim _{\left|\boldsymbol{x}-\boldsymbol{y}_{j}\right| \rightarrow 0} 4 \pi\left|\boldsymbol{x}-\boldsymbol{y}_{j}\right| \psi_{\boldsymbol{\sigma}}(\boldsymbol{x}), \\
f_{j \sigma} & =\lim _{\left|\boldsymbol{x}-\boldsymbol{y}_{j}\right| \rightarrow 0}\left[\psi_{\boldsymbol{\sigma}}(\boldsymbol{x})-\frac{q_{j \boldsymbol{\sigma}}}{4 \pi\left|\boldsymbol{x}-\boldsymbol{y}_{j}\right|}\right], \\
A_{j \boldsymbol{\sigma}, j^{\prime} \boldsymbol{\sigma}^{\prime}} & =0 \text { if } j \neq j^{\prime}, \\
A_{j \boldsymbol{\sigma}, j \boldsymbol{\sigma}^{\prime}} & =0 \text { if } \sigma_{k} \neq \sigma_{k}^{\prime} \text { for some } k \neq j, \\
A_{j \boldsymbol{\sigma}, j \boldsymbol{\sigma}^{\prime}} & =a_{j \sigma_{j}, j \sigma_{j}^{\prime}} \text { otherwise, with } \\
a_{j \sigma_{j}, j \sigma_{j}^{\prime}} & =\beta \delta_{\sigma_{j}, \sigma_{j}^{\prime}}+\sigma_{j} i \rho\left(1-\delta_{\sigma_{j}, \sigma_{j}^{\prime}}\right) \text { with } \beta, \rho \in \mathbb{R} .
\end{aligned}
$$

Then $H^{A}$ is self-adjoint and its resolvent, $R^{A}(z)=\left(H^{A}-z\right)^{-1}$, is the finite rank perturbation of the free resolvent $R(z)$ given by

$$
R^{A}(z)=R(z)+\sum_{j \boldsymbol{\sigma}, j^{\prime} \boldsymbol{\sigma}^{\prime}}\left(\left(\Gamma_{\beta, \rho}(z)\right)^{-1}\right)_{j \boldsymbol{\sigma}, j^{\prime} \boldsymbol{\sigma}^{\prime}}\left\langle\Phi_{j^{\prime} \boldsymbol{\sigma}^{\prime}}^{\bar{z}}, \cdot\right\rangle \Phi_{j \boldsymbol{\sigma}}^{z}, \quad z \in \rho\left(H^{A}\right),
$$

where $\Gamma_{\beta, \rho}(z)$ is the $N 2^{N} \times N 2^{N}$ matrix defined as

$$
\Gamma_{\beta, \rho}(z)=\Gamma(z)+A,
$$

with

$$
\begin{array}{rlrl}
\Gamma(z)_{j \sigma, j^{\prime} \boldsymbol{\sigma}^{\prime}} & =0, & & \text { if } \boldsymbol{\sigma} \neq \boldsymbol{\sigma}^{\prime}, \\
\Gamma(z)_{j \sigma, j \boldsymbol{\sigma}} & =\sqrt{z-\boldsymbol{\alpha} \cdot \boldsymbol{\sigma}} /(4 \pi i), & \\
\Gamma(z)_{j \sigma, j^{\prime} \boldsymbol{\sigma}} & =-G^{z-\boldsymbol{\alpha} \cdot \boldsymbol{\sigma}}\left(\boldsymbol{y}_{j}-\boldsymbol{y}_{j^{\prime}}\right), & & \text { if } j \neq j^{\prime},
\end{array}
$$

and $A$ is the matrix defined in (3-19)-(3-21).

Some particular features in the definition of $H^{A}$ are noteworthy:

- For the sake of convenience, the domain and action of $H^{A}$ are represented using the complex number $z$ but one can check that the definition of the operator is independent of the choice of $z$. 
- Functions in the domain of $H^{A}$ are either singular or zero in each scattering center $\boldsymbol{y}_{j}, j=1,2, \ldots, N$. The "charges" $q$ 's are the coefficients of the singular part whereas the $f$ 's are the values assumed in the scattering centers by the "regular part" of functions in the domain (i.e., the function minus its singular part in that point). The relation $\sum_{j^{\prime} \sigma^{\prime}} A_{j \sigma, j^{\prime} \sigma^{\prime}} q_{j^{\prime} \sigma^{\prime}}=f_{j \sigma}$ (see (3-15)) is then a singular boundary condition satisfied by the functions in the domain in each point $\boldsymbol{y}_{i}$. Relation (3-19) guarantees that the boundary condition satisfied in each point by functions in the domain do not depend on the boundary conditions satisfied elsewhere ("locality" of the self-adjoint extension). Notice that the matrix $A_{j \sigma, j^{\prime} \sigma^{\prime}}$ is defined in terms $\beta, \rho \in \mathbb{R}$ (see (3-21)), i.e., the two relevant parameters characterizing the interaction.

- The Hamiltonians one obtains for $\rho=0$ do not show any term indicating interaction between particle and spins. They correspond to point interaction Hamiltonians for the particle (see [Albeverio et al. 2005]) together with free evolution of the spins. Among the self-adjoint extensions of $\tilde{H}_{0}$ there are Hamiltonians where $\beta$ is taken as spin-dependent $(\beta(\sigma))$. Always in cases with $\rho=0$, the spins are still free, whereas the particle feels a point force in each $\boldsymbol{y}_{j}$ whose strength depends on the spin state in that same point. The latter were the Hamiltonians used to analyze neutron scattering by (fixed) nuclei.

- $\rho$ is the coupling constant of the particle-spin interaction. If $\rho$ is different from zero, the particle, in addition to the zero-range interaction with the points, can exchange energy with the spins. Condition (3-20) guarantees locality of the boundary conditions in this case.

- The spectrum of $H^{A}$ can have a very rich structure. In particular, several eigenstates embedded in the continuum when $\rho=0$ turn into resonances when $\rho \neq 0$ as a consequence of the interaction particle-spin. For our scope only the spectral structure at high energy will be relevant. It is easy to check that the spectrum of $H^{A}$ is purely continuous with no embedded eigenvalues for energies larger then $\max _{\sigma}(\boldsymbol{\alpha} \cdot \boldsymbol{\sigma})$.

The generalized eigenfunctions of $H^{A}$ are computable using the explicit form of the resolvent operator (3-22). For positive energies $\lambda>\max _{\sigma}(\boldsymbol{\alpha} \cdot \sigma)$ their $\sigma$ components are

$$
\begin{aligned}
& \varphi_{\beta, \rho}^{\boldsymbol{\sigma}}(\boldsymbol{x}, \lambda, \boldsymbol{\omega}) \otimes \mathcal{X}_{\boldsymbol{\sigma}}=\frac{(\lambda-\boldsymbol{\alpha} \cdot \boldsymbol{\sigma})^{1 / 4}}{(4 \pi)^{3 / 2}}\left[e^{i \sqrt{\lambda-\boldsymbol{\alpha} \cdot \boldsymbol{\sigma}} \boldsymbol{\omega} \cdot \boldsymbol{x}} \otimes \mathcal{X}_{\boldsymbol{\sigma}}\right. \\
&\left.+\sum_{j, j^{\prime}, \boldsymbol{\sigma}^{\prime}}\left(\Gamma_{\beta, \rho}(\lambda)\right)_{j, \boldsymbol{\sigma}, j^{\prime} \boldsymbol{\sigma}^{\prime}}^{-1} e^{i \sqrt{\lambda-\boldsymbol{\alpha} \cdot \boldsymbol{\sigma} \omega \cdot \boldsymbol{y}_{j}}} G^{\lambda-\boldsymbol{\alpha} \cdot \boldsymbol{\sigma}^{\prime}}\left(\boldsymbol{x}-\boldsymbol{y}_{j^{\prime}}\right) \otimes \mathcal{X}_{\boldsymbol{\sigma}^{\prime}}\right]
\end{aligned}
$$


where $\omega \in S^{2}$ is the unit vector representing the momentum direction. In fact, (3-25) remains valid for all values of $\lambda$ provided that the imaginary part of $\sqrt{\lambda-\boldsymbol{\alpha} \cdot \boldsymbol{\sigma}}$ is chosen positive for all $\sigma$ such that $\lambda-\boldsymbol{\alpha} \cdot \sigma<0$.

We consider the normalized initial condition

$$
\Psi^{0}=\psi_{0} \otimes \mathcal{X}_{\sigma_{0}},
$$

where $\sigma_{0}$ is the spin configuration in which $\sigma_{j}=-1$ for all $j$,

$$
\psi_{0}(\boldsymbol{x})=\hat{\mathcal{N}} \frac{e^{-x^{2} /\left(2 \gamma^{2}\right)}}{x} \sin \left(P_{0} x\right),
$$

with $x=|\boldsymbol{x}|$ and

$$
\hat{\mathcal{N}}=\frac{1}{\pi^{3 / 4} \sqrt{\gamma}\left(1-e^{-P_{0}^{2} \gamma^{2}}\right)^{1 / 2}},
$$

is a normalization factor making the norm of $\Psi^{0}$ equal to one. In momentum space the initial particle wave packet reads

$$
\hat{\psi}_{0}(\boldsymbol{k})=\hat{\mathcal{N}}^{\prime} / k\left[e^{-\left(\gamma^{2} / 2\right)\left(k-P_{0}\right)^{2}}-e^{-\left(\gamma^{2} / 2\right)\left(k+P_{0}\right)^{2}}\right]
$$

where $k=|\boldsymbol{k}|$ and $\hat{\mathcal{N}}^{\prime}=\gamma / 2 \hat{\mathcal{N}}$.

The meaning of the choice made above relates to the physical model: the spins are initially in the lowest energy state and are ready to detect the particle position during time evolution; the particle is propagating isotropically from the origin, with initial average radial momentum $P_{0}$ (corresponding to a wavelength $1 / P_{0}$ ). The parameter $\gamma$ represents the radial spread of the initial wave packet.

For simplicity, we fix $\alpha_{j}=\alpha>0$, for all $j$ in the Hamiltonian $H^{A}$ of the system. In this way $2 \alpha$ is the energy needed to flip the $j$-th spin from the down $\left(\sigma_{j}=-1\right)$ to the up state $\left(\sigma_{j}=1\right)$.

We consider situations in which the spin positions $y_{j}$ are distributed uniformly on a portion $\Sigma$ of linear dimension $D$ of a sphere of radius $L$ centered at the origin.

We assume that $\gamma \ll L$ in such a way that the initial wave packet is concentrated around the origin far from the scattering centers.

The parameter $\rho$ in $H^{A}$ has the dimension of inverse length and the role of the strength of the coupling between the particle and the spins; $1 / \beta$ is the scattering length of the scatterers.

We assume that the following inequalities between the order of magnitudes of relevant physical parameters hold:

$$
\begin{gathered}
1 / \beta \ll 1 / P_{0}<D, \\
\alpha \ll P_{0}^{2} \text { specifically } P_{0}^{2} \gtrsim N \alpha .
\end{gathered}
$$

The meaning of the assumptions above can be summarized as follows: 
The average initial energy of the particle is larger than the energy needed to produce any number of spin flips and the particle de Broglie wavelength is much larger than the scattering length of the point scatterers. The relation $1 / P_{0}<D$ will imply that two spherical waves

$$
\frac{\sin P_{0}\left|\boldsymbol{x}-\boldsymbol{y}_{j}\right|}{4 \pi\left|\boldsymbol{x}-\boldsymbol{y}_{j}\right|}, \quad j=j_{1}, j_{2},
$$

centered in two scatterers will show constructive interference only on the axis of the segment connecting the two points.

With the assumptions made above, Mott's conjecture can be rephrased as follows: consider the cone $C_{\Sigma}$ with apex at the origin and intersecting $S^{2}$ in $\Sigma$. Let us denote with $\chi_{\Sigma}$ the characteristic function of the cone. It is convenient to write the initial condition as the following superposition state

$$
\hat{\psi}_{0}(\boldsymbol{k})=\left(1-\chi_{\Sigma}(\boldsymbol{k})\right) \hat{\psi}_{0}(\boldsymbol{k})+\chi_{\Sigma}(\boldsymbol{k}) \hat{\psi}_{0}(\boldsymbol{k}) .
$$

We can show that the first term in (3-31) evolves almost freely giving a negligible contribution to any change in the configuration of spins in $\Sigma$. Only the second term in (3-31) can produce relevant changes in the spin configuration in $\Sigma$.

The precise statement will be formulated in terms of the long term behavior of the whole system (particle and spins). This requires the use of standard techniques in time-dependent and independent scattering theory (see [Reed and Simon 1979]). The wave operators $\Omega_{ \pm}$associated to the couple $H^{A}, H_{0}$ are defined as

$$
\begin{aligned}
& \Psi_{+}=\Omega_{+} \Psi=s-\lim _{t \rightarrow \infty} e^{i t H^{A}} e^{-i t H_{0}} \Psi, \\
& \Psi_{-}=\Omega_{-} \Psi=s-\lim _{t \rightarrow-\infty} e^{i t H^{A}} e^{-i t H_{0}} \Psi .
\end{aligned}
$$

As a consequence of the fact that the resolvent of $H^{A}$ is a finite rank perturbation of the resolvent of $H_{0}$, the wave operators exist and are complete. In particular one has

$$
\lim _{t \rightarrow \infty}\left\|e^{-i t H^{A}} \Psi-e^{-i t H_{0}} \Omega_{+}^{-1} \Psi\right\|_{\mathcal{H}}=0 .
$$

This means that $\Omega_{+}^{-1}$ applied to the initial state gives a modified initial state whose free evolution approximates the long term evolution of the system. In terms of $\Omega_{+}^{-1}$ our main result can be stated as follows:

Theorem 3.2. For any $\sigma \neq \sigma_{0}$, under the assumptions (3-30),

$$
\left\|\left(\Omega_{+}^{-1} \hat{\psi}^{0}\right)_{\sigma}(\boldsymbol{k})-\chi_{\Sigma}(\boldsymbol{k}) \hat{\psi}_{0}(\boldsymbol{k})\right\|_{L^{2}\left(\mathbb{R}^{3}\right)}<e^{-C P_{0}},
$$

where $C$ is a positive real constant and, as an abuse of notation, we use the same symbol $\Omega_{+}^{-1}$ for the wave operator acting on the particle Fourier space. 
Notice that the free evolution of the approximate modified initial state, denoted by $\chi_{\Sigma}(\boldsymbol{k}) \hat{\psi}_{0}(\boldsymbol{k})$, in (3-35) is characterized by a particle momentum always concentrated inside the cone and by an unaltered spin configuration. The result then states the following (conceivable) result: when the particle initial energy is very large compared to the energy necessary to flip a large number of spins

(1) the evolution of the particle state is not affected significantly by the interaction with the environment, and

(2) spins in a particular direction with respect to the source have negligible probability to be flipped by components of the initial wave packet heading in other directions.

The main steps heading to the result are sketched below. Only the proof of the main technical lemma will be detailed in the following.

The relation between wave operators and generalized eigenfunctions implies that the modified initial condition in a specific spin configuration reads

$$
\left(\Psi_{+}^{0}\right)_{\sigma}:=\left(\Omega_{+}^{-1} \Psi^{0}\right)_{\sigma}=\int_{\alpha \cdot \sigma}^{\infty} \mathrm{d} \lambda \int_{S^{2}} \mathrm{~d} \omega \phi_{\sigma}(\cdot, \lambda, \omega)\left(\varphi_{\beta, \rho}^{\sigma}(\lambda, \omega), \psi_{\sigma}^{0}\right),
$$

where

$$
\phi_{\sigma}(\boldsymbol{x}, \lambda, \omega)=\frac{(\lambda-\alpha \cdot \sigma)^{1 / 4}}{(4 \pi)^{3 / 2}} e^{i \sqrt{\lambda-\alpha \cdot \sigma} \omega \cdot \boldsymbol{x}}
$$

- The first step in the proof consists in carrying out an explicit computation of the scalar product of the initial conditions with the generalized eigenfunctions appearing in the integral on the right hand side of (3-36). If $\boldsymbol{y}$ is the position of a spin in $\Sigma$ and $\sigma \neq \sigma_{0}$ then

\section{Lemma 3.1.}

$$
\left\|\left(G^{\lambda-\widehat{\alpha \cdot \sigma}(\cdot-y)}, \hat{\psi_{0}}\right)-\left(G^{\lambda-\widehat{\alpha \cdot \sigma}(\cdot-y)}, \chi_{\Sigma} \hat{\psi}_{0}\right)\right\|<e^{C^{\prime} P_{0}}
$$

where $C^{\prime}$ is a positive real constant.

Proof. Let us write

$$
\begin{aligned}
F_{\boldsymbol{\sigma}, \Sigma}(\lambda,|\boldsymbol{y}|) \equiv\left(\left(G^{\lambda-\widehat{\boldsymbol{\alpha \cdot \sigma}(\cdot}}-\boldsymbol{y}\right), \chi_{\Sigma} \hat{\psi}_{0}\right) \\
=\int_{\mathbb{R}^{3}} \mathrm{~d} \boldsymbol{k} \overline{G^{\lambda-\widehat{\boldsymbol{\alpha} \cdot \boldsymbol{\sigma}}(\cdot-\boldsymbol{y})}} \hat{\psi}_{0}(\boldsymbol{k}) \chi_{\Sigma}(\boldsymbol{k})
\end{aligned}
$$


for the specific initial state of interest (3-27). The spherical wave Fourier transform is the distribution

$$
\begin{aligned}
\left.G^{\lambda-\widehat{\boldsymbol{\alpha} \cdot \boldsymbol{\sigma}}(\cdot-}-\boldsymbol{y}\right)=\frac{1}{(2 \pi)^{3 / 2}} \int_{\mathbb{R}^{3}} e^{-i \boldsymbol{k} \cdot \boldsymbol{x}} \frac{e^{i \sqrt{\lambda-\boldsymbol{\alpha} \cdot \boldsymbol{\sigma}}|\boldsymbol{x}-\boldsymbol{y}|}}{4 \pi|\boldsymbol{x}-\boldsymbol{y}|} \mathrm{d} \boldsymbol{x} \\
\quad=\frac{e^{-i \boldsymbol{k} \cdot \boldsymbol{y}}}{(2 \pi)^{3 / 2} k} \int_{0}^{\infty} \sin (k \xi) e^{i \sqrt{\lambda-\boldsymbol{\alpha} \cdot \boldsymbol{\sigma}} \xi} \mathrm{d} \xi,
\end{aligned}
$$

where $\sigma$ is a generic spin configuration $\sigma \neq \sigma_{0}$.

First, we compute explicitly the integral with respect to the momentum modulus $\mathrm{k}$. Neglecting terms exponentially decreasing faster then $e^{-c P_{0}^{2}}$ for any $c>\gamma^{2} / 2$, we perform the gaussian integral

$$
I(\xi) \equiv \int_{0}^{\infty} e^{i k|y| \cos \theta_{k, y}} \sin (k \xi) e^{-\gamma / 2\left(k-P_{0}\right)^{2}} \mathrm{~d} k=I_{+}(\xi)-I_{-}(\xi)
$$

where

$$
I_{ \pm}(\xi) \equiv \frac{1}{2 i} \int_{0}^{\infty} e^{i k|y| \cos \theta_{k, y}} e^{ \pm i k \xi} e^{-\gamma / 2\left(k-P_{0}\right)^{2}} \mathrm{~d} k
$$

and $\theta_{\boldsymbol{k}, \boldsymbol{y}}$ is the angle between the vectors $\boldsymbol{k}$ and $\boldsymbol{y}$. The result of the integration reads

$$
I_{ \pm}(\xi)=1 /(2 i \gamma) \sqrt{\pi / 2} e^{-\gamma^{2} / 2 P_{0}^{2}} e^{-1 /(2 \gamma 2) z_{ \pm}^{2}} \operatorname{Erfc}\left(-i /(\sqrt{2} \gamma) z_{ \pm}\right),
$$

where $z_{ \pm}= \pm \xi+|\boldsymbol{y}| \cos \theta_{\boldsymbol{k}, \boldsymbol{y}}-i \gamma^{2} P_{0}$ and Erfc denotes the complementary error function.

Defining $b=\sqrt{\lambda+N \alpha}$, we have

$$
\begin{aligned}
F_{\boldsymbol{\sigma}, \Sigma}(\lambda,|\boldsymbol{y}|) & \\
= & \frac{1}{(2 \pi)^{3 / 2}} \hat{\mathcal{N}}^{\prime} \int \chi_{\Sigma}\left[\int_{0}^{\infty}\left(I_{+}(\xi)-I_{-}(\xi)\right) e^{-i b \xi} \mathrm{d} \xi\right] \mathrm{d} \Sigma \\
= & D e^{-\left(b-P_{0}\right)^{2} \gamma^{2} / 2} \int_{S^{2}} \chi_{\Sigma}(\boldsymbol{k}) e^{i P_{0}|\boldsymbol{y}| \cos \theta_{\boldsymbol{k}, \boldsymbol{y}}} e^{-|\boldsymbol{y}|^{2} /\left(2 \gamma^{2}\right) \cos ^{2} \theta_{\boldsymbol{k}, \boldsymbol{y}}} \\
& {\left[\int_{0}^{\infty} e^{-\left(\xi /(\sqrt{2} \gamma)+i\left(b-P_{0}\right) \gamma / \sqrt{2}\right)^{2}} e^{-\xi / \gamma^{2}|\boldsymbol{y}| \cos \theta_{\boldsymbol{k}, \boldsymbol{y}}} \operatorname{Erfc}\left(-i /(\sqrt{2} \gamma) z_{+}\right) \mathrm{d} \xi\right] \mathrm{d} \Sigma } \\
+ & D e^{-\left(b+P_{0}\right)^{2} \gamma^{2} / 2} \int_{S^{2}} \chi_{\Sigma}(\boldsymbol{k}) e^{i P_{0}|\boldsymbol{y}| \cos \theta_{\boldsymbol{k}, \boldsymbol{y}}} e^{-|\boldsymbol{y}|^{2} /\left(2 \gamma^{2}\right) \cos ^{2} \theta_{\boldsymbol{k}, \boldsymbol{y}}} \\
& {\left[\int_{0}^{\infty} e^{-\left(\xi /(\sqrt{2} \gamma)+i\left(b+P_{0}\right) \gamma / \sqrt{2}\right)^{2}} e^{+\xi / \gamma^{2}|\boldsymbol{y}| \cos \theta_{\boldsymbol{k}, \boldsymbol{y}}} \operatorname{Erfc}\left(-i /(\sqrt{2} \gamma) z_{-}\right) \mathrm{d} \xi\right] \mathrm{d} \Sigma, }
\end{aligned}
$$


where $D=1 /(2 \pi)^{3 / 2} \hat{\mathcal{N}}^{\prime} 1 /(2 i \gamma) \sqrt{\pi / 2}$. Being the integral in the variable $\xi$ uniformly bounded for any finite value of $b, P_{0}$ and $\theta_{\boldsymbol{k}, \boldsymbol{y}}$, it is possible to integrate over the momentum directions which lie inside the cone $\mathcal{C}_{\Sigma}$.

In order to give an estimate for large $P_{0}|\boldsymbol{y}|$ of the integral we will make use of a stationary phase argument. We will take the axis of the cone to be the polar axis $\theta_{\boldsymbol{k}}=0$. Both integrals in (3-39) have the form

$$
\int_{0}^{\theta_{\max }} e^{i P_{0}|\boldsymbol{y}| \cos \theta_{\boldsymbol{k}, \boldsymbol{y}}} G\left(\cos \theta_{\boldsymbol{k}, \boldsymbol{y}}\right) \sin \theta_{\boldsymbol{k}} \mathrm{d} \theta_{\boldsymbol{k}} \mathrm{d} \phi_{\boldsymbol{k}},
$$

with $\cos \theta_{\boldsymbol{k}, \boldsymbol{y}}=\cos \theta_{\boldsymbol{k}} \cos \theta_{\boldsymbol{y}}+\sin \theta_{\boldsymbol{k}} \sin \theta_{\boldsymbol{y}} \cos \left(\phi_{\boldsymbol{k}}-\phi_{\boldsymbol{y}}\right)$.

It is easy to check that the phase $\cos \theta_{\boldsymbol{k}, \boldsymbol{y}}$ is stationary only if $\theta_{\boldsymbol{k}}=\theta_{\boldsymbol{y}}$ and $\phi_{k}=\phi_{y}$ and that the Hessian matrix determinant computed at the point of stationary phase is $\sin ^{2} \theta_{\boldsymbol{y}}$. We deduce that the case of stationary phase applies only if $\boldsymbol{y} /|\boldsymbol{y}|$ belongs to the cone $\mathcal{C}_{\Sigma}$ and that, in such a case, the leading term of (3-40) reads

$$
\frac{2 \pi}{i} \frac{e^{i P_{0}|\boldsymbol{y}|}}{P_{0}|\boldsymbol{y}|} G\left(\cos \theta_{\boldsymbol{k}, \boldsymbol{y}}=1\right) \frac{\sin \theta_{\boldsymbol{y}}}{\left|\sin \theta_{\boldsymbol{y}}\right|} .
$$

If $y$ is inside the cone $\mathcal{C}_{\Sigma}$ and $\theta_{\max }<\pi / 2$, we then have

$$
\begin{aligned}
F_{\boldsymbol{\sigma}, \Sigma}(\lambda,|\boldsymbol{y}|) \simeq- & \frac{\hat{\mathcal{N}}}{8} \frac{e^{i P_{0}|\boldsymbol{y}|}}{P_{0}|\boldsymbol{y}|} e^{-\left(b-P_{0}\right)^{2} \gamma^{2} / 2} e^{-|\boldsymbol{y}|^{2} /\left(2 \gamma^{2}\right)} \\
& {\left[\int_{0}^{\infty} e^{-\left(\xi /(\sqrt{2} \gamma)+i\left(b-P_{0}\right) \gamma / \sqrt{2}\right)^{2}} e^{-\xi / \gamma^{2}|\boldsymbol{y}|} \operatorname{Erfc}\left(-i /(\sqrt{2} \gamma) v_{+}\right) \mathrm{d} \xi\right] } \\
- & \frac{\hat{\mathcal{N}}}{8} \frac{e^{i P_{0}|\boldsymbol{y}|}}{P_{0}|\boldsymbol{y}|} e^{-\left(b+P_{0}\right)^{2} \gamma^{2} / 2} e^{-|\boldsymbol{y}|^{2} /\left(2 \gamma^{2}\right)} \\
& {\left[\int_{0}^{\infty} e^{-\left(\xi /(\sqrt{2} \gamma)+i\left(b+P_{0}\right) \gamma / \sqrt{2}\right)^{2}} e^{+\xi / \gamma^{2}|\boldsymbol{y}|} \operatorname{Erfc}\left(-i /(\sqrt{2} \gamma) v_{-}\right) \mathrm{d} \xi\right], }
\end{aligned}
$$

where $v_{ \pm}= \pm \xi+|\boldsymbol{y}|-i \gamma^{2} P_{0}$.

If the scatterer position lies outside the cone, standard results in approximation of integrals in the nonstationary case proves the exponential decay stated in the lemma.

Notice that, as a by-product, the lemma proves that the main contribution to the integral with respect to $\lambda$ in (3-36) is attained for $b \approx P_{0}$ which, under our assumption, means $\sqrt{\lambda} \approx P_{0}$.

- As a second step, one has to examine the coefficients in the sum of spherical waves $G^{\lambda-\alpha \cdot \sigma}$ in (3-25). In particular, it is necessary to check that the matrix $\Gamma_{\beta, \rho}(\lambda)$ is invertible and to control the norm of its inverse. Under the assumptions of the dynamical and geometrical parameters of the system 
listed above it is always possible to find a $\beta$, independent on the number of spins, for which the matrix $\Gamma_{\beta, \rho}(\lambda)$ is invertible and the norm of its inverse is bounded uniformly in the number of spins. Details about the invertibility and the continuum limit of the inverse of the $\Gamma$ matrix are given in [Figari and Teta 2014] (see also [Figari et al. 1988] for a similar result in the case of point interactions without spin dependence).

Theorem 3.2 specifies where the main contribution to the flipping probability comes from, yet does not give quantitative indications about the transition probabilities to any specific final configuration of the environment. In order to investigate thoroughly the asymptotic behavior of the particle and the environment and characterize its dependence on the number of flipped spins, it is necessary to go one step further:

- Prove a kind of Huygen's principle stating that the spherical waves centered in a large number of points uniformly distributed on $\Sigma$ interfere constructively only inside the cone $C_{\Sigma}$. The situation is analogous to the case of electromagnetic wave propagation through a hole when the linear dimensions of the hole are much larger than the wavelength (absence of diffraction).

- Give an estimate of the probability that a significant fraction of spins turns out to be flipped.

These two last technical steps, requiring detailed combinatorial estimates, will be examined in a paper in preparation.

To conclude, we want to summarize what we obtained in the language of the environment induced decoherence: if a large number of "clouds" of spin dependent point scatterers are present on the sphere of radius $L$, the above stated result amounts to saying that the long term behavior of the whole system state is the incoherent sum of states with support in distant regions of the configuration space, each characterized by a sharply defined particle momentum direction and a significant change in the spin configuration only relative to spins in that specific direction.

\section{References}

[Adami et al. 2004] R. Adami, R. Figari, D. Finco, and A. Teta, "On the asymptotic behaviour of a quantum two-body system in the small mass ratio limit", J. Phys. A 37:30 (2004), 7567-7580.

[Adami et al. 2006] R. Adami, R. Figari, D. Finco, and A. Teta, "On the asymptotic dynamics of a quantum system composed by heavy and light particles", Comm. Math. Phys. 268:3 (2006), 819852.

[Albeverio et al. 2005] S. Albeverio, F. Gesztesy, R. Høegh-Krohn, and H. Holden, Solvable models in quantum mechanics, 2nd ed., AMS Chelsea Publishing, Providence, RI, 2005.

[Bell 1987] J. S. Bell, Speakable and unspeakable in quantum mechanics, Cambridge University Press, 1987. 
[Blanchard et al. 2000] P. Blanchard, D. Giulini, E. Joos, C. Kiefer, and I.-O. Stamatescu (editors), Decoherence: theoretical, experimental and conceptual problems, Lecture Notes in Physics 538, Springer, 2000.

[Bohr 1928] N. Bohr, "The quantum postulate and the recent development of atomic theory", Nature, London 121 (1928), 580-590.

[Born 1926] M. Born, “Zur Quantenmechanik der Stoßvorgänge”, Z. Phys. 37:12 (1926), 863-867.

[Born and Jordan 1925] M. Born and P. Jordan, "Zur Quantenmechanik", Z. Phys. 34:1 (1925), $858-888$.

[Cacciapuoti et al. 2007] C. Cacciapuoti, R. Carlone, and R. Figari, "Spin-dependent point potentials in one and three dimensions", J. Phys. A 40:2 (2007), 249-261.

[Condon and Gurney 1928] E. Condon and R. Gurney, "Wave mechanics and radioactive disintegration", Nature 122 (1928), 439.

[Dell'Antonio et al. 2008] G. Dell'Antonio, R. Figari, and A. Teta, "Joint excitation probability for two harmonic oscillators in one dimension and the Mott problem", J. Math. Phys. 49:4 (2008), 042105, 20.

[Dell'Antonio et al. 2010] G. Dell'Antonio, R. Figari, and A. Teta, "A time-dependent perturbative analysis for a quantum particle in a cloud chamber", Ann. Henri Poincaré 11:3 (2010), 539-564.

[Figari and Teta 2013] R. Figari and A. Teta, "Emergence of classical trajectories in quantum systems: the cloud chamber problem in the analysis of Mott (1929)", Archive for History of Exact Sciences 67:2 (2013), 215-234.

[Figari and Teta 2014] R. Figari and A. Teta, Quantum dynamics of a particle in a tracking chamber, Springer Briefs in Physics, Springer, 2014.

[Figari et al. 1988] R. Figari, H. Holden, and A. Teta, "A law of large numbers and a central limit theorem for the Schrödinger operator with zero-range potentials", J. Statist. Phys. 51:1-2 (1988), $205-214$.

[Finco and Teta 2011] D. Finco and A. Teta, "Asymptotic expansion for the wave function in a one-dimensional model of inelastic interaction", J. Math. Phys. 52:2 (2011), 022103, 19.

[Gamow 1928] G. Gamow, "Quantum theory of atomic nucleus”, Z. Phys. 51:204 (1928).

[Gupta and Ghosh 1946] N. N. D. Gupta and S. K. Ghosh, "A report on the Wilson cloud chamber and its applications in physics", Rev. Mod. Phys. 18 (1946), 225-290.

[Hornberger 2009] K. Hornberger, "Introduction to decoherence theory", pp. 221-276 in Entanglement and decoherence, edited by A. Buchleitner et al., Lecture Notes in Phys. 768, Springer, Berlin, 2009.

[Joos et al. 2003] E. Joos, H. D. Zeh, C. Kiefer, D. Giulini, J. Kupsch, and I.-O. Stamatescu, Decoherence and the appearance of a classical world in quantum theory, 2nd ed., Springer, Berlin, 2003.

[Leone and Robotti 2004] M. Leone and N. Robotti, "A note on the Wilson cloud chamber (1912)", Eur. J. Phys. 25:6 (2004), 781.

[Mott 1929] N. F. Mott, "The wave mechanics of $\alpha$-ray tracks", Proc. R. Soc. Lond., Ser. A 126 (1929), 79-84.

[von Neumann 1932] J. von Neumann, Mathematische Grundlagen der Quantenmechanik, Grundlehren der math. Wiss. 38, Springer, Berlin, 1932. Translated as Mathematical Foundations of Quantum Mechanics, Princeton University Press, 1955.

[Recchia and Teta 2014] C. Recchia and A. Teta, "Semiclassical wave-packets emerging from interaction with an environment", J. Math. Phys. 55:1 (2014), 012104, 24. 
[Reed and Simon 1979] M. Reed and B. Simon, Methods of modern mathematical physics III, Academic Press, New York, 1979.

[Schrödinger 1982] E. Schrödinger, Collected papers on wave mechanics, Chelsea, New York, 1982.

[Teta 2010] A. Teta, "Classical behaviour in quantum systems: the case of straight tracks in a cloud chamber”, Eur. J. Phys. 31:1 (2010), 215.

Received 4 May 2016. Revised 13 Jun 2016. Accepted 1 Aug 2016.

RODOLFO FIGARI: figari@na.infn.it

Dept. of Physics "Ettore Pancini" and National Institute of Nuclear Physics,

University of Napoli Federico II, Complesso Univ. Monte Sant'Angelo Ed. 6, Via Cintia,

I-80126 Napoli, Italy

Alessandro Teta: teta@mat.uniroma1.it

Dipartimento di Matematica G. Castelnuovo, Sapienza Università di Roma, P.le A. Moro 5, I-00185 Roma, Italy 
EDITORIAL BOARD

ANTONIO CARCATERRA

ERIC A. CARLEN

FRANCESCO DELL'ISOLA

RAFFAELE ESPOSITO

ALBERT FANNJIANG

Gilles A. FranCFORT

Pierangelo MARCATI

JEAN-JACQUES MARIGO

PETER A. MARKOWICH

MARTIN OSTOJA-STARZEWSKI

PIERRE SEPPECHER

DAVID J. STEIGMANN

PAUl STEINMANN

PierRe M. SuQueT

MANAGING EDITORS

MICOL AMAR

CORRADO LATTANZIO

ANGELA MADEO

MARTIN OSTOJA-STARZEWSKI

ADVISORY BOARD

ADNAN AKAY

Holm AltenBaCH

MICOL AMAR

HARM ASKES

TEODOR ATANACKOVIĆ

VICTOR BERDICHEVSKY

GUY BOUCHITTÉ

ANDREA BRAIDES

ROBERTO CAMASSA

MAURO CARFORE

ERIC DARVE

FELIX DARVE

ANNA DE MASI

GianPiEtro DEL Piero

EMMANUELE Di BENEDETTO

BERNOLD FIEDLER

IRENE M. GAMBA

DAVID Y. GAO

SERGEY GAVRILYUK

TIMOTHY J. HEALEY

DOMINIQUE JEULIN

ROGER E. KHAYAT

CORRADO LATTANZIO

ROBERT P. LIPTON

ANGELO LUONGO

ANGELA MADEO

JUAN J. MANFREDI

CARLO MARCHIORO

GÉRARD A. MAUGIN

ROBERTO NATALINI PATRIZIO NEFF

ANDREY PIATNITSKI

ERRICO PRESUTTI

MARIO PULVIRENTI

LUCIO RUSSO

Miguel A. F. SANJUAN

PATRICK SElVADURAI

ALEXANDER P. SEYRANIAN

MIROSLAV ŠILHAVÝ

GUIDO SWEERS

ANTOINETTE TORDESILLAS

LEV TRUSKINOVSKY

JUAN J. L. VELÁZQUEZ VINCENZO VESPRI ANGELO VULPIANI msp.org/memocs

Università di Roma "La Sapienza", Italia

Rutgers University, USA

(CO-CHAIR) Università di Roma "La Sapienza", Italia

(TREASURER) Università dell'Aquila, Italia

University of California at Davis, USA

(CO-CHAIR) Université Paris-Nord, France

Università dell'Aquila, Italy

École Polytechnique, France

DAMTP Cambridge, UK, and University of Vienna, Austria

(CHAIR MANAGING EDITOR) Univ. of Illinois at Urbana-Champaign, USA

Université du Sud Toulon-Var, France

University of California at Berkeley, USA

Universität Erlangen-Nürnberg, Germany

LMA CNRS Marseille, France

Università di Roma "La Sapienza", Italia

Università dell'Aquila, Italy

Université de Lyon-INSA (Institut National des Sciences Appliquées), France

(CHAIR MANAGING EDITOR) Univ. of Illinois at Urbana-Champaign, USA

Carnegie Mellon University, USA, and Bilkent University, Turkey

Otto-von-Guericke-Universität Magdeburg, Germany

Università di Roma "La Sapienza", Italia

University of Sheffield, UK

University of Novi Sad, Serbia

Wayne State University, USA

Université du Sud Toulon-Var, France

Università di Roma Tor Vergata, Italia

University of North Carolina at Chapel Hill, USA

Università di Pavia, Italia

Stanford University, USA

Institut Polytechnique de Grenoble, France

Università dell'Aquila, Italia

Università di Ferrara and International Research Center MEMOCS, Italia

Vanderbilt University, USA

Freie Universität Berlin, Germany

University of Texas at Austin, USA

Federation University and Australian National University, Australia

Université Aix-Marseille, France

Cornell University, USA

École des Mines, France

University of Western Ontario, Canada

Università dell' Aquila, Italy

Louisiana State University, USA

Università dell'Aquila, Italia

Université de Lyon-INSA (Institut National des Sciences Appliquées), France University of Pittsburgh, USA

Università di Roma "La Sapienza”, Italia

Université Paris VI, France

Istituto per le Applicazioni del Calcolo "M. Picone", Italy

Universität Duisburg-Essen, Germany

Narvik University College, Norway, Russia

Università di Roma Tor Vergata, Italy

Università di Roma "La Sapienza”, Italia

Università di Roma “Tor Vergata”, Italia

Universidad Rey Juan Carlos, Madrid, Spain

McGill University, Canada

Moscow State Lomonosov University, Russia

Academy of Sciences of the Czech Republic

Universität zu Köln, Germany

University of Melbourne, Australia

École Polytechnique, France

Bonn University, Germany

Università di Firenze, Italia

Università di Roma La Sapienza, Italia

MEMOCS (ISSN 2325-3444 electronic, 2326-7186 printed) is a journal of the International Research Center for the Mathematics and Mechanics of Complex Systems at the Università dell'Aquila, Italy.

Cover image: "Tangle” by $\odot$ John Horigan; produced using the Context Free program (contextfreeart.org).

PUBLISHED BY

7 mathematical sciences publishers

nonprofit scientific publishing

http://msp.org/

(C) 2016 Mathematical Sciences Publishers 
Special issue in honor of

Lucio Russo

Lucio Russo: A multifaceted life

Raffaele Esposito and Francesco dell'Isola

The work of Lucio Russo on percolation Geoffrey R. Grimmett

"Mathematics" and "physics" in the science of harmonics Stefano Isola

From quantum to classical world: emergence of trajectories in a quantum system Rodolfo Figari and Alessandro Teta

Propagation of chaos and effective equations in kinetic theory: a brief survey Mario Pulvirenti and Sergio Simonella

What decides the direction of a current? Christian Maes

A remark on eigenvalue perturbation theory at vanishing isolation distance Fiorella Barone and Sandro Graffi

Some results on the asymptotic behavior of finite connection probabilities in percolation Massimo Campanino and Michele Gianfelice

Correlation inequalities for the Potts model Geoffrey R. Grimmett

Quantum mechanics: some basic techniques for some basic models, I: The models Vincenzo Grecchi

Quantum mechanics: some basic techniques for some basic models, II: The techniques Vincenzo Grecchi

On stochastic distributions and currents

Vincenzo Capasso and Franco Flandoli

A note on Gibbs and Markov random fields with constraints and their moments Alberto Gandolfi and Pietro Lenarda

Quantum mechanics: light and shadows (ontological problems and epistemic solutions) Gianfausto Dell'Antonio

MEMOCS is a journal of the International Research Center for the Mathematics and Mechanics of Complex Systems at the Università dell' Aquila, Italy.

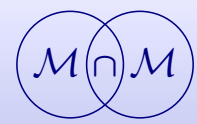

